\title{
Serum Sickness Following Equine Rabies Immunoglobulin Administration - A Case Study
}

\author{
Swetaleena Ashe', Dipanweeta Routray ${ }^{2}$, Tapas Ranjan Behera ${ }^{3}$ \\ ${ }^{1}$ Junior Resident, ${ }^{2,3}$ Assistant Professor, Department of Community Medicine, SCB Medical College and Hospital, Cuttack, \\ Odisha, India.
}

DOI: https://doi.org/10.24321/0019.5138.201929

I $\quad \mathbf{N} \quad \mathbf{F} \quad \mathbf{O}$

\section{Corresponding Author:}

Dipanweeta Routray, Department of Community Medicine, SCB Medical College and Hospital, Cuttack, Odisha, India.

E-mail Id:

drdipanweeta@gmail.com

Orcid Id:

https://orcid.org/0000-0002-9212-3651

How to cite this article:

Ashe S, Routray D, Behera TR. Serum Sickness Following Equine Rabies Immunoglobulin Administration - A Case Study. J Commun Dis 2019; 51(3): 77-79.

Date of Submission: 2019-08-02

Date of Acceptance: 2019-10-18
$\begin{array}{llllllll}\mathbf{A} & \mathbf{B} & \mathbf{S} & \mathbf{T} & \mathbf{R} & \mathbf{A} & \mathbf{C} & \mathbf{T}\end{array}$

A fourteen years old male child presented to the Anti-Rabies Clinic of a tertiary care hospital with rashes all over the body associated with myalgia and mild fever following treatment with Anti-Rabies vaccine and Equine Rabies Immunoglobulin (ERIG) for Category III monkey bite injury. On tenth day of vaccination, the child developed generalized rashes all over the body. He was primarily treated with anti-histaminics and analgesics. However, the rashes still increased in size and later involved the whole body including the face. He was then treated with corticosteroids (Injection Dexamethasone $4 \mathrm{mg} / 2 \mathrm{~mL}$ ) and was completely cured after three days. The adverse reaction following ERIG administration was diagnosed as a case of Serum Sickness and reported to the Adverse Drug Reaction Monitoring Centre of the College. The purpose of this case study is to create awareness about the adverse drug reactions following ERIG administration among the health care personnel, importance of prompt management of such Serum Sickness cases and timely reporting to the ADR Monitoring Centre. It has its own implications for policy makers.

Keywords: Serum Sickness, Equine Rabies Immunoglobulin, Adverse Drug Reaction Monitoring, Rabies, Anti-Rabies Vaccination

\section{Introduction}

Rabies is a zoonotic disease caused by Lyssavirus. It mainly affects the CNS and cause fatal encephalomyelitis in humans and animals. It is a $100 \%$ fatal disease and hence deserves utmost importance in slightest of injury from animal bite/ scratch; else a small negligence could cost a human life. This deadly disease can be prevented with just timely and appropriate anti rabies prophylaxis. ${ }^{1}$ Modern medical science with its fast evolution has gifted mankind with products from animals which are life-saving for humans. One such is the Equine Rabies Immunoglobulin (ERIG) which is administered to humans upon any bite/ scratch from warm-blooded animal. Heterologous serum administration is associated with a number of adverse effects. So, skin sensitivity test is performed in order to know the clinical safety of the immunoglobulin. Howsoever, as the sensitivity and specificity of skin sensitivity is very low, so testing will be of little help in predicting anaphylaxis or serum sickness. ${ }^{2} \mathrm{WHO}$ even recommends that there is no scientific background for performing a skin test before administration of ERIG in the present era as the current available ERIG are pure. Serum sickness in humans is a reaction to proteins in antiserum derived from a nonhuman animal source, occurring 5-10 days after exposure. It is a type III immune complex hypersensitivity disorder. It usually presents with symptoms such as rashes, arthralgia, 
fever, malaise, headache and gastrointestinal symptoms. ${ }^{3}$ The human immune system mistakenly treats the serum proteins as antigen and start producing antibodies, which form immune complexes and precipitate in the walls of the blood vessels. Proper treatment with anti-histaminics, analgesics and corticosteroids, would completely cure the patient else it could lead to as serious a condition as shock to cause death of the patient. ${ }^{4}$

\section{Case Profile}

A fourteen years old male child from Cuttack, Odisha presented to the Anti-Rabies Clinic (ARC) of a teaching hospital in Central Odisha on $15^{\text {th }}$ of May 2019 with the chief complaints of eruption of rashes all over the body for one day. It was associated with mild degree of fever, malaise and generalized pruritus. The child had a history of Category III monkey scratch over the right forearm on $6^{\text {th }}$ of May. On $3^{\text {rd }}$ of May, the Cyclone FONI in Odisha caused devastation and as an aftermath to it many monkeys lost their houses due to fall of large number of trees. They turned out to be aggressive and thus many cases of monkey bite were reported at the ARC during this time.

His mother washed the wound with soap and water and he was immediately accompanied by his father to the ARC where he was administered with Inj. Tetanus Toxoid $0.5 \mathrm{~mL}$ Intramuscular, Injection Purified Vero Cell Rabies Vaccine (PVRV) (B No 18 URAB050 manufactured by Human Biologicals Institute), $0.1 \mathrm{~mL}$ in both arms intradermally. He weighed $49 \mathrm{Kg}$ and hence was advised to be infiltrated with Equine Rabies Immunoglobulin (ERIG) (B No. 191049 manufactured by Premium Serums and Vaccines Pvt. Ltd.) 1900 IU i.e. $6.5 \mathrm{~mL}$ after the skin sensitivity test. The test was done using $0.1 \mathrm{~mL}$ of ERIG diluted with $0.9 \mathrm{~mL}$ of normal saline provided by the manufacturer over forearm. He was observed for any induration but only a wheal of 7 $\mathrm{mm}$ was seen. Hence, he was administered, $5 \mathrm{~mL}$ ERIG at the site of bite and rest $1.5 \mathrm{~mL}$ was administered at the antero lateral aspect of left thigh, intra muscularly. He was further advised with Tablet Levocetirizine 1 tablet SOS in empty stomach with Tablet Paracetamol SOS and to attend the ARC for completion of rest PVRV doses as scheduled on $9^{\text {th }}$ May, $13^{\text {th }}$ May and $3^{\text {rd }}$ June.

The child was apparently alright from the initiation of treatment but on $15^{\text {th }}$ of May i.e. (10 $10^{\text {th }}$ day of treatment) he presented to the ARC with rashes all over the body. The rashes first appeared on the upper limbs followed by legs, abdomen and back and they gradually increased in size. It was associated with mild degree of fever, malaise, and generalized pruritus. He denied of having any previous allergy to any food items or drugs. The child was then diagnosed to be a case of Serum Sickness following ERIG administration.

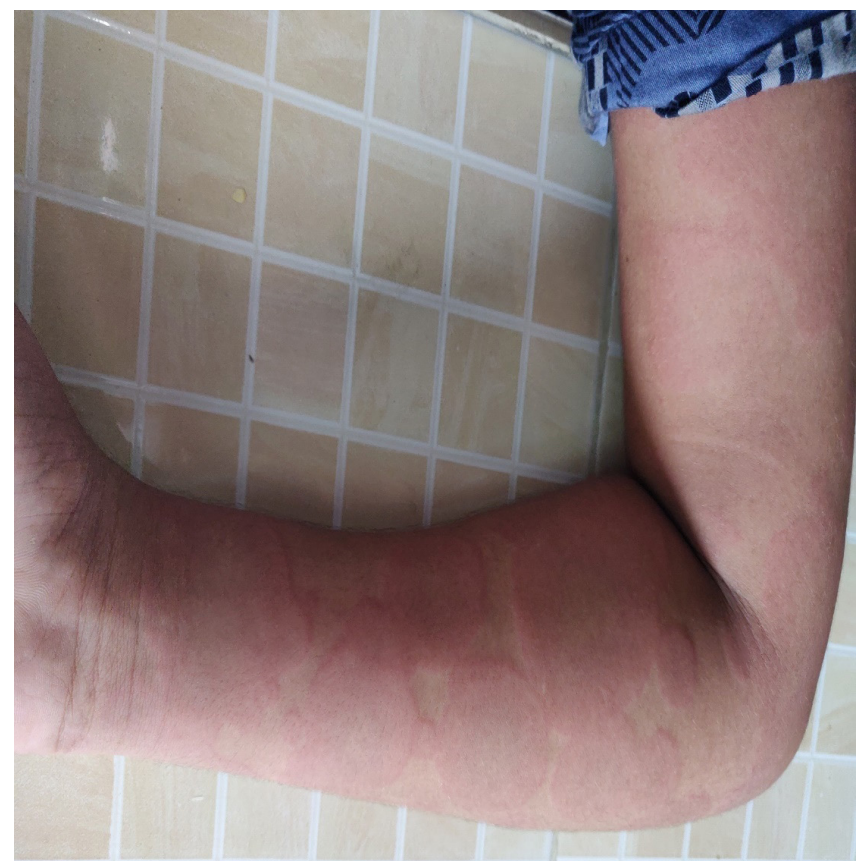

Figure I.Rashes on the forearm due to serum sickness On examination, vitals were stable. The child was advised to take Fexofenadine $180 \mathrm{mg}$ three times daily after food and tablet Paracetamol 500mg twice daily for next five days. Again, on next day i.e. on $16^{\text {th }}$ May the child reported to the ARC with increased size and number of the rashes and now involving face. He was then immediately administered Injection Pheniramine maleate $0.5 \mathrm{~mL}$ intramuscularly and Injection Dexamethasone $4 \mathrm{mg} / 2 \mathrm{~mL}$ by intravenous route. Second opinion was sought from the Department of Pediatrics, where he was advised the same medication to continue. The child was then followed up over telephone and he reported decrease in the size of rashes by evening. He was called after a day for clinical evaluation and was found to be completely cured of the rashes. He was further advised to complete his last scheduled Anti-Rabies Vaccine (ARV) on $3^{\text {rd }}$ June without any fear which he had completed. This case was reported to the Adverse Drug Reaction monitoring Centre of the institution.

\section{Discussion}

In our study, the patient was a male child of 14 years of age. Children aged less than 15 years are prone to animal bites as evidenced by other studies such as one by Satpathy DM et al. ${ }^{5}$ at Berhampur and Mohanty $S$ et al. at Cuttack $^{6}$ in Odisha who found that $62.5 \%$ and $35.94 \%$ cases respectively attending the ARC were under 15 years of age. Male children are at more risk of animal bites probably due to their outdoor playing habits. ${ }^{7}$ The children being more playful and less defensive are more vulnerable for such bites. Cases attending ARC with history of monkey bite/ scratches are usually less as compared to dog bites/ scratches. A study reported by Ashwath Narayana DH et 
al. ${ }^{8}$ found $2.1 \%$ of biting animals comprising of monkeys. However, dogs are a major cause of rabies as also assessed by a national multicenter survey where dogs (96.2\%) were mainly responsible for human rabies deaths. ${ }^{9}$ We noticed a good practice in the parent of child i.e. washing the wound immediately with soap and water. A study on "Effectiveness of child to child method of education regarding Rabies and its prevention" by Shwetha et al. ${ }^{10}$ showed only $21.5 \%$ of subjects knew of washing wound with soap and water prior to education which was followed by $100 \%$ of them knowing it after education. Serum sickness is a rare complication occurring in response to ERIG administration. Among the local adverse effects cited in Tripathy RM et al. ${ }^{2}$, urticaria, dizziness, redness, vomiting, localized swelling and headache were common. However, in our case there was no such symptom on the day of initiation of treatment. The child complained of various systemic adverse effects such as generalized pruritus, malaise, mild fever and rashes. He was diagnosed to have Serum Sickness as also found in other studies by Behera TR et al. in Odisha and Wilde et al. in Bangkok where the incidences were 3\% and 1.6\% respectively. ${ }^{11,12}$ Study by Maharana $S$ et al. ${ }^{13}$ found rashes to be the most common symptom in $97.82 \%$ of cases following ERIG administration in the same setting which was similar to our case. Our case reported of rashes on tenth day after administration of ERIG which was a rare occurrence as only $12 \%$ of cases following ERIG infiltration reported symptoms of serum sickness on tenth day..$^{13}$ Only one case among 48 cases observed for serum sickness by Maharana S et al. ${ }^{13}$ required corticosteroids, however this patient regardless of being treated with antihistaminic still needed corticosteroids for management.

\section{Conclusion}

Reactions following immunoglobulin such as Serum sickness though a rare adverse effect yet awareness regarding its symptoms among the patients and proper guidelines to manage such cases is of utmost importance. Adverse effects following immunization has been a hindrance for successful outcome of any immunization program. So, it is very necessary to make people aware of different adverse effects that could follow vaccination and create a sense of urgency to seek timely treatment. It is also recommended that all immunization centers to be fully equipped with Adverse Effects Following Immunization (AEFI) kits or anaphylaxis kits as well as protocols for management of such cases. The life-saving drugs should be properly labelled, its usage made very clear to all the staff dealing with vaccination. In this era, where immunization has been a great step in averting disease and deaths, AEFI must be properly managed and reported to ADRM Centers on regular basis. This can surely be a step, safe enough, towards achieving the goal of "Dog-mediated Human Rabies free India by 2030 ".

\section{Acknowledgment}

We highly appreciate the cooperation of the child and his father for having understood the purpose of reporting and giving a detailed history along with cooperating for the follow up.

\section{Conflict of Interest: None \\ References}

1. National Guidelines for Rabies Prophylaxis and Intradermal Administration of Cell Culture Rabies Vaccines. National Institute of Communicable Diseases. 2007.

2. Tripathy RM, Satpathy SP, Das S. Outcome of skin sensitivity test for assessing adverse reactions to equine rabies immunoglobulin in a tertiary setup of South Odisha. APCRI Journal 2018; 19(2): 13-15.

3. Yerushalmi J, Zvulunov A, Halevy S. Serum Sickness-like reactions. Cutis 2002; 69(5): 395-397.

4. Serum sickness. Available from: http://en.m.wikipedia. org/wiki/Serum_sickness.

5. Satapathy DM, Sahu T, Behera TR et al. Socio clinical profile of rabies cases in anti-rabies clinic, MKCG Medical College Orissa. Indian Journal of Public Health 2005; 49(4): 241-242.

6. Mohanty S, Behera TR, Pattnaik N. Clinical safety of an equine rabies immunoglobulin, APCRI Journal 2018; 19(2): 9-11.

7. Mohammad K, Gupta P. A study of human rabies cases admitted in infectious disease hospital KGMU, Lucknow, North India. Int J Community Med Public Health 2018; 5(11): 4795-4798.

8. Ashwath Narayana DH, Ravish HS, Ramesh Holla. Clinical evaluation of safety of Equine Rabies immunoglobulin. APCRI Journal 2011; 12(2): 12-15.

9. Sudarshan MK. Assessing burden of rabies in India: WHO sponsored National multicentric Rabies survey, 2003. Indian Journal of Community Medicine 2005; 30(3): 100-101.

10. Shwetha, Vinay M, Harish Br. Effectiveness of child to child method of Education regarding Rabies and its prevention. APCRI Journal 2018; 19(2): 28-29.

11. Behera TR, Satapathy DM, Pratap AK et al. Postexposure prophylaxis for rabies with ERIG and IDRV in children. J Commun Dis 2011; 43(1): 31-37.

12. Wilde $\mathrm{H}$, Chomchey $\mathrm{P}$, Punyaratabandhu P. Purified equine rabies immune globulin: A safe and affordable alternative to human rabies immunoglobulin. Bulletin of the WHO 1989; 67(6): 731-736.

13. Maharana S, Behera TR, Pattnaik N. Serum sickness in patients receiving equine rabies immunoglobulin. J Commun Dis. 2018; 50(2): 1-4. 